\title{
Correction to: SATO: An Efficient Propositional Prover
}

\section{Hantao Zhang}

\section{Correction to:}

\section{Chapter 28 in: SATO: An Efficient Propositional Prover}

Hantao Zhang

The original version of Chapter 28 was inadvertently published with incorrect word in the title "prepositional" instead of "propositional". The word has been corrected. 